\title{
Anthropogenic impact on the air environment condition of megalopolis and suburban areas of forestland
}

\author{
Vadim Rogov ${ }^{1}$, Nataliya $\mathrm{Kravchenko}^{2}$, Oksana Bagaeva $^{1}$, Anastasiya Guseva ${ }^{3}$, and Evgenii \\ Sugak $^{1}$ \\ ${ }^{1}$ Reshetnev SibSU, Life Safety Department, Krasnoyarsk, Russia \\ ${ }^{2}$ Reshetnev SibSU, Construction Materials Engineering and Wood Science Department, Krasnoyarsk, \\ Russia \\ ${ }^{3}$ ReshetnevSibSU, Foreign Language Department, Krasnoyarsk, Russia
}

\begin{abstract}
The anthropogenic impact of the megalopolis on the release of volatile matters by woody plants, air ionization in the suburban areas of the forestland for the period 1998 - 2018 was monitored. A decrease in the emission of volatile matters by woody plants, negative ions, an increase in positive ions was revealed. The composition of the air environment of a megalopolis for a long time was studied. It has been established that anthropogenic factors (dust content, gas contamination, electromagnetic radiation) increase the amount of positive ions by order of magnitude. It was revealed that the presence of electromagnetic radiation significantly affects the physical properties of air. The effect of ionization and volatile matters on the deposition rate of suspended particles was determined in laboratory conditions. Theoretical studies have been carried out, on the basis of which deterministic, differential equations of the effect of ionization on dust content, gas contamination of the air environment and the interaction of air ionization with polydisperse dust, volatile substances to produce adsorbate and its deposition rate have been obtained. The data of experimental and theoretical studies made it possible to develop measures to reduce anthropogenic environmental factors, which will improve the ecological situation and reduce the incidence of megalopolis residents.
\end{abstract}

\section{Introduction}

Suburban and park areas are the lungs of a metropolis, affecting the cleaning of its air environment. A positive effect is achieved due to the release of volatile matters and negative ions. One of the reasons for the increase in the incidence of the inhabitants of the metropolis is its anthropogenic impact, which has a negative effect on the physical and chemical properties of the air environment of the suburban areas of the forestland.

Staying in the forest mobilizes the body's defenses and enhances its immunobiological functions. Volatile plant emissions contribute to the accumulation of vitamin $\mathrm{C}$ and other biologically active substances in it [1]. There is a significant amount of negative ions in the 
forest air. Inhalation of ionized air leads to decrease in sugar and increase in oxygen in the blood, increases the activity of enzymes.

Until the present, studies and monitoring of the influence of anthropogenic factors on the emission of volatile matters and natural air ionization by woody plants have not been conducted. The monitoring results showed decrease in volatile matters by woody plants and increase in the amount of positive air ions due to urbanization and the growth of anthropogenic factors.

The aim of the work is to study the influence of anthropogenic impact on the release of volatile matters and natural air ionization in the suburban areas of the forestland, their interaction. The paper studies the physical and chemical properties of forest air - the presence of negative and positive ions, the release of volatile matters by woody plants. The studies were conducted during the period 1998-2018. These studies allow us to determine the influence of the anthropogenic impact of the metropolis on the tropobiosphere of coniferous forest.

Theoretical dependences of the effect of natural ionization on the concentration of dust and the pathogenic microflora of the air environment were obtained.

A comparative analysis of published materials on the emission of volatile matters by woody plants was carried out, as well as measurements of the natural ionization of air in the forestland and megalopolis: the amount of negative and positive ions. $[1,2,3,4]$.

\section{Monitoring of the environment}

The dynamics of changes in the quantitative content of negative and positive ions in the suburban areas of the forestland during the period 1998-2018 is presented in Table 1. Air ionization was measured using the ion counter device in the warm period of the year (in July), since this period is characterized by the largest amount of volatile matters emissions and an increased content of negative ions [6].

Table 1. Dynamics of changes in the quantitative content of negative and positive ions during the period 1998-2018 in the suburban areas of the forestland

\begin{tabular}{|c|c|c|c|c|c|}
\hline Period & 1998 & 2003 & 2008 & 2013 & 2018 \\
\hline The amount of negative ions, $\mathrm{e} / \mathrm{cm}^{3}$ & 10000 & 9000 & 9000 & 9200 & 7000 \\
\hline The amount of positive ions, $\mathrm{e} / \mathrm{cm}^{3}$ & 50 & 150 & 200 & 300 & 350 \\
\hline
\end{tabular}

From table 1 it is seen that over the indicated period in the suburban areas of the forestland, the number of negative ions decreased by $30 \%$, the amount of positive ions increased by 7 times.

The objectives of the study also included studying the composition of the air environment in a metropolis. Data on the amount of negative and positive ions in the air environment of a megalopolis (Central and Kirov districts of Krasnoyarsk) are presented in Table 2.

Analysis of the data in table 2 shows that the amount of positive ions exceeds the amount of negative ions in the studied areas of the city. During this period, there is a significant increase in the amount of positive ions and a decrease in negative ones.

Table 2. Dynamics of changes in the quantitative content of negative and positive ions during the period 2016-2019 in Krasnoyarsk (warm season) 


\begin{tabular}{|l|l|l|l|l|}
\hline \multicolumn{1}{|c|}{ Наименование/period } & \multicolumn{1}{|c|}{2016} & \multicolumn{1}{|c|}{2017} & 2018 & 2019 \\
\hline $\begin{array}{l}\text { The amount of negative ions, e/cm } \\
\text { district) }\end{array}$ & $\begin{array}{l}500- \\
600\end{array}$ & $\begin{array}{l}500- \\
600\end{array}$ & $\begin{array}{l}300- \\
350\end{array}$ & 300 \\
\hline $\begin{array}{l}\text { The amount of positive ions, e/cm } \\
\text { district) }\end{array}$ & $\begin{array}{l}700- \\
800\end{array}$ & $\begin{array}{l}1100- \\
1200\end{array}$ & 1900 & 2200 \\
\hline $\begin{array}{l}\text { The amount of negative ions, e/cm } \\
\text { district) }\end{array}$ & 1100 & 1100 & 900 & $\begin{array}{l}850- \\
900\end{array}$ \\
\hline $\begin{array}{l}\text { The amount of positive ions, e/cm } \\
\text { district) }\end{array}$ & 900 & 1200 & $\begin{array}{l}1800- \\
1900\end{array}$ & 2100 \\
\hline
\end{tabular}

According to the observations of the authors, electromagnetic radiation (EMR), which is found in almost all districts of the city, has a negative effect on the inhabitants of the city and the air environment. The presence of electromagnetic radiation negatively affects the physical properties of the air environment, increasing the amount of positive ions by an order of magnitude compared to negative ones, forming clusters of dust and gases [5].

The authors' studies established that the air in forestland and meadows contains from 2,000 to 10,000 negative aero ions in $\mathrm{cm}^{3}\left(\mathrm{e} / \mathrm{cm}^{3}\right)$, and in residential buildings their concentration decreases to $25 \mathrm{e} / \mathrm{cm}^{3}$, and in residential districts of the city it does not exceed $500 \mathrm{e} / \mathrm{cm}^{3}$ or is completely absent and an excess of positive ions is observed, which adversely affects the health of the population of the metropolis.

Based on the studies and the state report on the condition and environmental protection in the Krasnoyarsk Territory during 2016-2018 the data were obtained which are presented in table 3.

Table 3. The dynamics of the content of harmful substances in the air of a megalopolis during 20162018

\begin{tabular}{|l|l|l|l|l|l|l|l|}
\hline & $\begin{array}{l}\text { Suspen- } \\
\text { ded } \\
\text { matters }\end{array}$ & $\begin{array}{l}\text { Sulfur } \\
\text { dioxide }\end{array}$ & $\begin{array}{l}\text { Carbon } \\
\text { oxide }\end{array}$ & $\begin{array}{l}\text { Nitrogen } \\
\text { dioxid }\end{array}$ & $\begin{array}{l}\text { Nitrogen } \\
\text { oxide }\end{array}$ & $\begin{array}{l}\text { Hydrogen } \\
\text { sulfide }\end{array}$ & $\begin{array}{l}\text { Benza- } \\
\text { pyrene }\end{array}$ \\
\hline 2016 & $0,75(4,8)$ & $(3,0)$ & $\begin{array}{l}0,4 \\
(1,8)\end{array}$ & $\begin{array}{l}0,85 \\
(1,15)\end{array}$ & $0,4(-)$ & $(15,1)$ & $5,1(40,6)$ \\
\hline 2017 & $0,85(6,0)$ & $\begin{array}{l}0,26 \\
(3,4)\end{array}$ & $\begin{array}{l}0,45 \\
(3,0)\end{array}$ & $\begin{array}{l}0,75 \\
(1,21)\end{array}$ & $\begin{array}{l}0,35 \\
(1,01)\end{array}$ & $(14,1)$ & $5,3(40,1)$ \\
\hline 2018 & $0,89(7,6)$ & $\begin{array}{l}0,28 \\
(4,02)\end{array}$ & $\begin{array}{l}0,47 \\
(3,6)\end{array}$ & $0,9(1,5)$ & $\begin{array}{l}0,25 \\
(1,79)\end{array}$ & $(14,8)$ & $\begin{array}{l}6,5 \\
(50,6)\end{array}$ \\
\hline
\end{tabular}

During the studied period there is an increase in suspended particles and harmful substances. In authors' opinion, this is due to an increase in the number of automobile transport in the metropolis.

\section{Experimental research}

To determine the effect of negative ions on the content of dust and gas in the air environment, original studies were conducted in laboratory conditions. A laboratory setup was developed and mounted, which includes a source with control of the amount of negative ions and biologically active substance (BAS). BAS was a light fraction of essential oils of woody plants (spruce, pine, cedar, juniper, forbs). The results are presented in Figure 1.

The figure shows the time dependences $(t, m)$ of the concentration of suspended dust particles $\left(C, \mathrm{mg} / \mathrm{m}^{3}\right)$ in air without additives (curve 1), in an air-ionized environment (curve 3 ), and with an admixture of volatile productions (curves 2 and 4 ). 


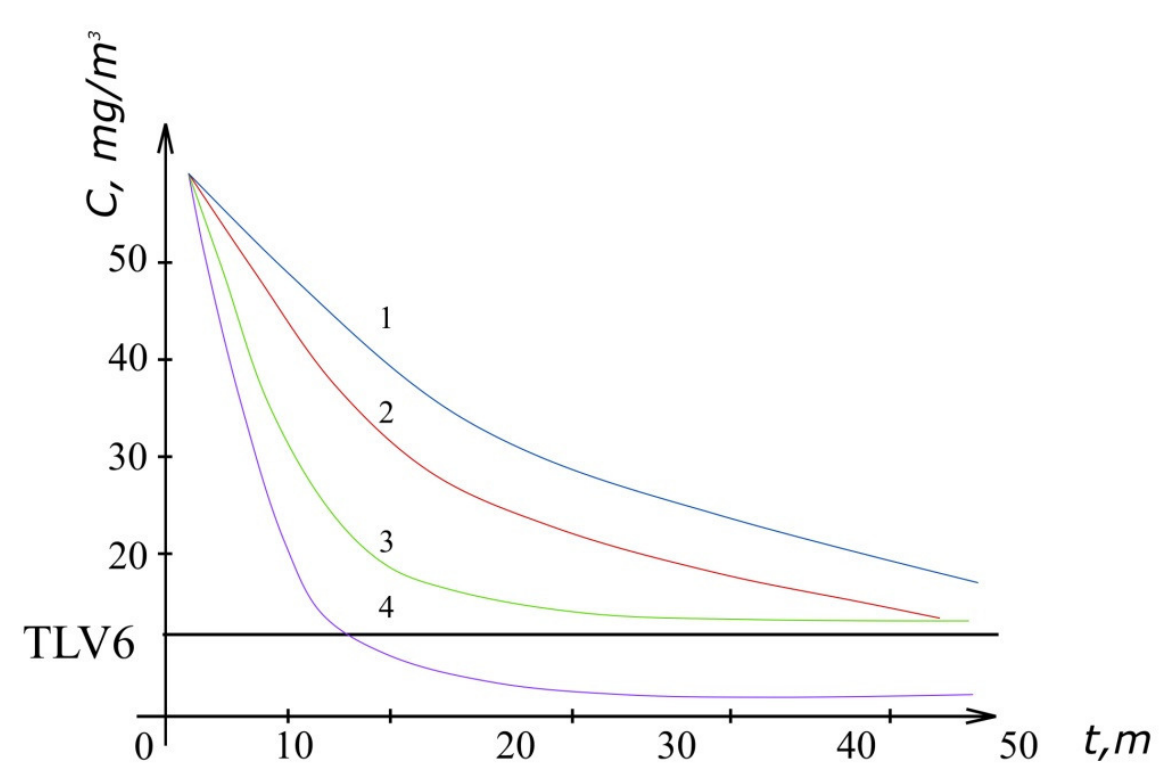

dependence of dust concentration on the type of air treatment:

1 - natural dust deposition;

2 - under the influence of volatile substances;

3 - under the influence of ionization;

4 - under the influence of ionization and volatile substances.

Studies have shown that negative ions in combination with volatile substances have an effective influence on reducing of dust content and gas contamination of the air environment.

\section{Theoretical description of the process}

To describe the impact of anthropogenic factors on air ionization, a theoretical study was carried out and mathematical dependences were obtained.

The model equilibrium state of a layer of adsorbed ions on the particle surface is described by the Fokker-Planck equation[6]:

$$
\frac{\partial p}{\partial t}=\frac{\partial}{\partial x_{i}}\left(i_{i} p-\frac{\partial}{\partial x_{i}} D_{\mathrm{ij}} p\right)
$$

where: $x_{i}=x, x_{2}=y, x_{3}=z$ are the coordinates of the point in the volume field of the particle-adsorbate conglomerate containing the particle-medium interface, $l_{i}$ are the projections of the mobility vector of adsorbed ions corresponding to them, $p$ is the charge distribution density; $D_{i j}$ is the tensor of their diffusion coefficients.

After converting the Fokker-Planck equation (1), taking into account the properties of the adsorbate material (dust particles), we obtain the equation[6]: 


$$
\frac{\partial p}{\partial t}=D \frac{\partial^{2} p}{\partial r^{2}}+\alpha \frac{\partial U}{\partial r} \frac{\partial p}{\partial r}
$$

where $r$ is the reduced radius of the adsorbate, $\alpha$ is the mobility vector.

The interaction of air ions with the volatile substances of woody plants affects the structure of the adsorbate with the formation of polydispersed moments with the further aggregation of aerosol solid particles and their deposition from the air environment.

If it contains only water and ions, the adsorbent-adsorbate interface will coincide with the active surface of the aerosol particles. If the medium contains a high molecular weight additive, for example, volatile matters, a layer such as an adhesive coating will eventually be formed on the surface of the particle. In the first case, the total mass of particles is limited in time, the mass of the adsorbate will increase to an equilibrium state. Then, due to an increase in molecular exchange with the medium, the outer interface of the adsorbate is eroded, and its mass ceases to increase over time. In the second case, the thickness of the adhesive film is not limited in time. The particle becomes two-layer. Moreover, such a conglomerate structure will determine the nature of the adsorption of water and ions. The adhesive film may be hydrophobic, which completely excludes the adsorption of water. In the case of its hydrophilicity, the formation of a third layer of water and ions is also possible.

In a practical sense, the mechanism of deposition of aerosol particles is represented by translational degrees of freedom. In this case, we mean polydisperse particles suspended in air, because otherwise, the probability of collision of particles moving in the same direction is low. The aerosol mixture on the average particle size scale is generally heterogeneous, that is, there are density fluctuations and randomly directed microflows in the medium (convection, thermal diffusion, diffusion). Particles of high dispersion are carried away by microflows and are pushed by density fluctuations.

All of the above leads to the random movement of particles in the Brownian picture. Moreover, as it is easy to show, the Stokes law is its determining factor [6]:

$$
\mathrm{FC}=6 \pi \eta \mathrm{rv}_{4}
$$

where $F c$ is the force of viscous friction, $\quad \eta$ is the dynamic viscosity of the medium, $r$ is the radius of the particle, $v_{4}$ is its velocity.

To precipitate the obtained adsorbate in air environment under the influence of ionization and volatile matters, the particle motion in the field of gravity was considered. At the initial moment $\mathrm{t}=0$ a spherical particle with radius $r$ moves under the action of gravity in a viscous medium with a velocity $v_{0}$. Two opposing forces act on it: the gravity $m g$ and Stokes $6 \pi \eta \mathrm{rV}_{4}$. The resulting or acting force $m g-6 \pi \eta \mathrm{rv}_{4}$ according to the d'Alembert principle is equal to the product of mass and acceleration:

$$
m v_{u}=m g-6 \pi \eta r v_{u}
$$

After transformations of equation (4), we obtain the final expression for determining the deposition rate of adsorbate particles $V_{0 c}$, which is determined by the formula

$$
v_{0 c}=\frac{\mathrm{mg}}{6 \pi \eta r} .
$$


The obtained mathematical dependences explain the mechanism of interaction of air ionization with polydispersed dust, volatile matters to produce adsorbate and the rate of its deposition from the air environment

\section{Conclusions}

1. For the period from 1998 to 2018 , studies were conducted on the release of volatile matters by woody plants under the forest canopy in the suburban areas of the forestland (50 - $70 \mathrm{~km}$ from the metropolis). As a result it turned out that the amount of negative ions decreased over the indicated time period by $30 \%$, while the amount of positive ions increased sevenfold.

2. For the period from 2016 to 2019, studies of the air environment of the metropolis were conducted. An increasing dynamics of positive ions and a decrease in negative ions were revealed. The amount of suspended particles and harmful substances in the air increased due to an increase in road transport.

3. The effect of electromagnetic radiation on the increase in positive ions by an order of magnitude compared with negative ones has been established.

4. Studies have been carried out at the developed laboratory facility, which showed that negative ions in combination with volatile matters have an effective impact on reducing dust content and gas contamination of the air environment.

5. The obtained mathematical dependences provide a theoretical justification for the interaction of negative ions and volatile matters with polydisperse dust in the air.

Based on the theoretical and experimental studies, measures have been developed to reduce the anthropogenic impact on the air environment and residents of the megalopolis:

1. To create air with the content of negative ions up to $8000-10000 \mathrm{e} / \mathrm{cm}^{3}$ in the park zones of Krasnoyarsk.

2. Creation of an ecologically clean zone in parks and squares of Krasnoyarsk by planting phytoncide woody plants.

\section{References}

1. V.A. Rogov, R.A. Stepen, V.V. Kulak, Conifers of the Boreal Area: Theor. and Appl. Research J., 33, 168-170 (2015)

2. V.A. Rogov, A.V. Rogov, R.A. Stepen, Chemistry of plant raw materials, 4, $181-184$. (2008)

3. Luis A. Gurovich, Electrophysiology of Woody Plants. Electrophysiology - From Plants to Heart, Dr. Saeed Oraii (Ed.), InTech (2012)

4. Perveen, Shagufta, Chemical composition and antimicrobial activities of essential oils of some coniferous plants cultivated in Egyp. IJPR, 16 (Jan. 2017)

5. V.A. Prusakova, V.A. Rogov, V.V. Kulak, Forest and chemical complexes; problems and solutions, 1, $206-208$ (2015)

6. V.A. Rogov, Influence of negative ions and volatile terpenoids on the air purification of processing areas of woodworking enterprises (Publishing House of Moscow State University, 2002) 